\title{
B. Merkmale der Finanz- und Handelsströme zwischen der Schweiz und den Entwicklungsländern
}

Gérard Perroulaz, Xavier Tschumi Canosa and Tanja Guggenbühl

\section{OpenEdition}

\section{Journals}

Electronic version

URL: http://journals.openedition.org/sjep/90

DOI: $10.4000 /$ sjep.90

ISSN: 1663-9677

Publisher

Institut de hautes études internationales et du développement

\section{Printed version}

Date of publication: 1 avril 2008

Number of pages: 241-261

ISBN: 978-2-940415-02-1

ISSN: $1660-5926$

Electronic reference

Gérard Perroulaz, Xavier Tschumi Canosa und Tanja Guggenbühl, «B. Merkmale der Finanz- und Handelsströme zwischen der Schweiz und den Entwicklungsländern », Schweizerisches Jahrbuch für Entwicklungspolitik [Online], 27-1 | 2008, Online erschienen am: 22 Juni 2010, abgerufen am 07 September 2020. URL : http://journals.openedition.org/sjep/90 ; DOI : https://doi.org/10.4000/sjep.90 


\section{B. Merkmale der Finanz- und Handelsströme zwischen der Schweiz und den Entwicklungs- ländern}

\section{B.2 Aussenhandel der Schweiz}

\section{B.2.1 Übersicht über den Aussenhandel der Schweiz}

Die Aussenwirtschaftstätigkeit nimmt eine sehr bedeutende Stellung in der Wirtschaft der Schweiz ein. Ein von zwei Franken des Bruttoinlandsprodukts (BIP) stammt aus dem Export. 2006 beliefen sich die Gesamteinfuhren der Schweiz auf 165,4 Milliarden Franken und die Ausfuhren erreichten die Rekordzahl von 177,5 Milliarden Franken ${ }^{1} .2007$ erreichten die Importe mit 183,4 Milliarden Franken und die Exporte mit 197,3 Milliarden Franken neue Rekordhöhen ${ }^{2}$.

\section{$\square$ Entwicklung des Aussenhandels der Schweiz in den letzten Jahren}

\section{Grafik B.1: Entwicklung des Aussenhandels der Schweiz, 1990-2007 (in Millionen Franken)}

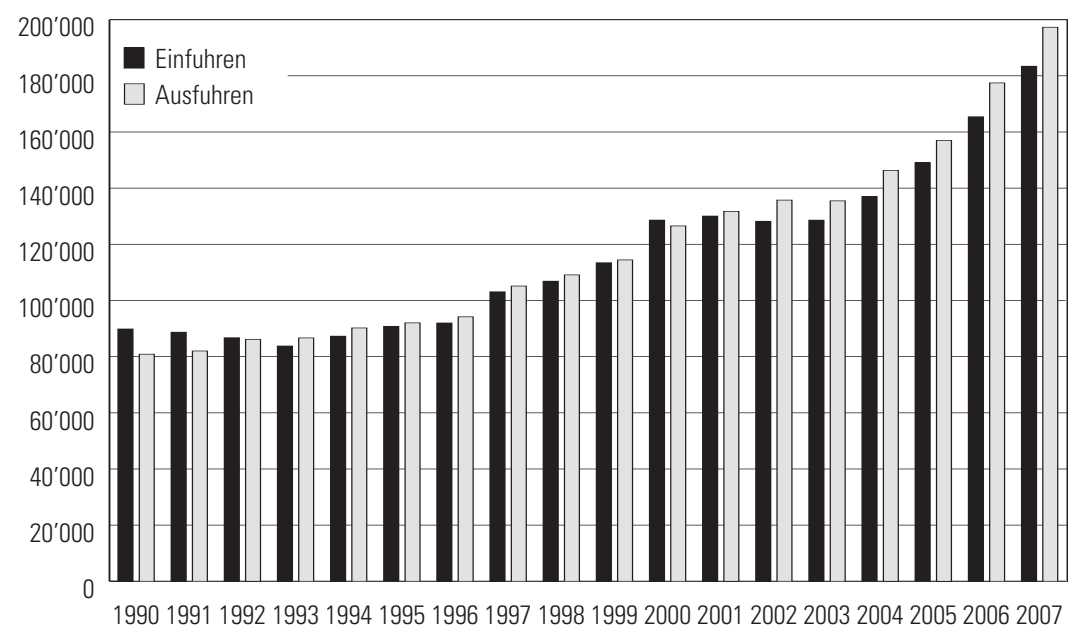

Quelle: Grafik erstellt anhand der Tabelle „Aussenhandel der Schweiz seit 1980 (Gesamtwert 1)” der Eidgenössischen Zollverwaltung, einsehbar unter http://www.ezv.admin.ch >Themen >Aussenhandelsstatistik >Provisorische Ergebnisse 2007. Anmerkung: Aussenhandel ohne Edelmetalle, Schmucksteine, Kunstgegenstände und Antiquitäten (Gesamtwert 1 der Zollstatistik). Der Goldhandel ist sogar aus der Zollstatistik ganz ausgeschlossen.

1 Ohne Edelmetalle, Schmucksteine, Kunstgegenstände und Antiquitäten (Gesamtwert 1 der Zollstatistik). Mit diesen Produkten beliefen sich die Ausfuhren der Schweiz 2006 auf 185,2 Milliarden Franken und die Einfuhren auf 177,1 Milliarden Franken.

2 Statistik der Eidgenössischen Zollverwaltung, Bern. 
Der Aussenhandel der Schweiz hat in den letzten Jahren einen sehr starken Zuwachs verzeichnet. Grafik B.1 zeigt die Entwicklung des Aussenhandels der Schweiz seit Anfang der 90er Jahre. Die Ausfuhren haben regelmässig zugenommen, wobei sie von 80,9 Milliarden Franken im Jahr 1990 auf 197,3 Milliarden Franken im Jahr 2007 anstiegen. Die Einfuhren haben - mit Ausnahme einer Stagnation von 1990 bis 1993 und eines Rückgangs zwischen 2001 und 2003 - ebenfalls regelmässig zugenommen. Von 2003 bis 2007 haben sich die Ein- und Ausfuhren mit einem Zuwachs von über 40 Prozent noch deutlicher erhöht.

Die Steigerung des Aussenhandels ist mit der Entwicklung des Welthandels vergleichbar, der namentlich wegen der zunehmenden Globalisierung eine starke Expansion verzeichnet. Die Einfuhren an Energieträgern haben zwischen 2005 und 2006 wertmässig um 25 Prozent zugenommen, was hauptsächlich auf die Erhöhung der Erdölpreise zurückzuführen ist.

\section{$\square$ Wichtigste Handelspartner der Schweiz}

Die in Tabelle B.1 aufgeführten 30 wichtigsten Handelspartner der Schweiz machten 2006 95,6 Prozent der Gesamteinfuhren und 89,4 Prozent der Gesamtausfuhren der Schweiz aus. Aus den Aussenhandelsdaten nach Ländern geht hervor, dass die Schweiz vor allem mit den Nachbarländern und den übrigen Industriestaaten in Europa und Übersee (USA, Japan, Kanada) Handelsbeziehungen unterhält. 2006 kamen ein Drittel der Schweizer Einfuhren aus Deutschland und 20 Prozent der gesamten Schweizer Ausfuhren gingen in dieses Land. Der Handel mit den Ländern der Europäischen Union machte 78,6 Prozent der Gesamteinfuhren und 61,6 Prozent der Gesamtausfuhren der Schweiz aus.

Ebenfalls aus Tabelle B.1 wird ersichtlich, dass 2006 lediglich sieben Entwicklungsländer unter den 30 wichtigsten Bezugsländern und sechs Entwicklungsländer unter den 30 wichtigsten Absatzländern der Schweiz aufgeführt waren. 
Tabelle B.1: Die 30 wichtigsten Handelspartner der Schweiz, 2006 (in Millionen Franken, Anteile und kumulierte Anteile in Prozent)

\begin{tabular}{|c|c|c|c|c|c|c|c|}
\hline \multicolumn{4}{|c|}{ Wichtigste Bezugsländer } & \multicolumn{4}{|c|}{ Wichtigste Absatzländer } \\
\hline \multirow[t]{2}{*}{ Land $^{\mathrm{a}}$} & \multicolumn{3}{|c|}{ Einfuhren } & \multirow[t]{2}{*}{ Land $^{\mathrm{a}}$} & \multicolumn{3}{|c|}{ Ausfuhren } \\
\hline & Mio Fr. & $\%^{\mathrm{b}}$ & $\Sigma \%^{\mathrm{c}}$ & & Mio Fr. & $\%^{\mathrm{b}}$ & $\Sigma \%^{\mathrm{c}}$ \\
\hline 1. Deutschland & $55^{\prime} 099.7$ & 33.3 & 33.3 & 1. Deutschland & $35^{\prime} 827.6$ & 20.2 & 20.2 \\
\hline 2. Italien & $18^{\prime} 426.0$ & 11.1 & 44.5 & 2. USA & $18^{\prime} 255.0$ & 10.3 & 30.5 \\
\hline 3. Frankreich & $17^{\prime} 096.7$ & 10.3 & 54.8 & 3. Italien & $15^{\prime} 913.8$ & 9.0 & 39.4 \\
\hline 4. USA & $88^{\prime} 308.4$ & 5.0 & 59.8 & 4. Frankreich & $15^{\prime} 224.7$ & 8.6 & 48.0 \\
\hline 5. Niederlande & $8^{\prime} 267.0$ & 5.0 & 64.8 & 5. Grossbritannien & $8^{\prime} 343.1$ & 4.7 & 52.7 \\
\hline 6. Österreich & 7'496.6 & 4.5 & 69.3 & 6. Spanien & $6^{\prime} 880.7$ & 3.9 & 56.6 \\
\hline 7. Grossbritannien & $6^{\prime} 006.0$ & 3.6 & 73.0 & 7. Japan & $6^{\prime} 361.0$ & 3.6 & 60.2 \\
\hline 8. Belgien & $5^{\prime} 093.3$ & 3.1 & 76.0 & 8. Niederlande & $6^{\prime} 034.1$ & 3.4 & 63.6 \\
\hline 9. Irland & 4'613.1 & 2.8 & 78.8 & 9. Österreich & $5 ' 829.1$ & 3.3 & 66.9 \\
\hline 10. Spanien & 4'038.4 & 2.4 & 81.3 & 10. China & $33^{\prime} 753.0$ & 2.1 & 69.0 \\
\hline 11. China & 3'918.6 & 2.4 & 83.6 & 11. Hongkong & $33^{\prime} 562.0$ & 2.0 & 71.0 \\
\hline 12. Japan & $2 ' 614.9$ & 1.6 & 85.2 & 12. Belgien & $33^{\prime} 053.1$ & 1.7 & 72.7 \\
\hline 13. Schweden & 1'757.9 & 1.1 & 86.3 & 13. Kanada & 2 '695.9 & 1.5 & 74.2 \\
\hline 14. Libyen & 1'677.2 & 1.0 & 87.3 & 14. Türkei & $2^{\prime} 301.9$ & 1.3 & 75.5 \\
\hline 15. Tschechische Rep. & 1'389.2 & 0.8 & 88.1 & 15. Russland & $2^{\prime} 224.5$ & 1.3 & 76.8 \\
\hline 16. Finnland & 1'192.2 & 0.7 & 88.9 & 16. Indien & 1'849.4 & 1.0 & 77.8 \\
\hline 17. Dänemark & 1'082.2 & 0.7 & 89.5 & 17. Singapur & 1'800.9 & 1.0 & 78.8 \\
\hline 18. Nigeria & 1'046.5 & 0.6 & 90.2 & 18. Schweden & 1'754.6 & 1.0 & 79.8 \\
\hline 19. Kanada & 966.0 & 0.6 & 90.7 & 19. Polen & 1'740.4 & 1.0 & 80.8 \\
\hline 20. Polen & 928.3 & 0.6 & 91.3 & 20. Verein. Arab. Emirate & 1'684.2 & 0.9 & 81.8 \\
\hline 21. Hongkong & 830.0 & 0.5 & 91.8 & 21. Südkorea & 1'666.0 & 0.9 & 82.7 \\
\hline 22. Ungarn & 775.1 & 0.5 & 92.3 & 22. Australien & 1'530.6 & 0.9 & 83.6 \\
\hline 23. Brasilien & 775.0 & 0.5 & 92.7 & 23. Brasilien & 1'458.5 & 0.8 & 84.4 \\
\hline 24. Südkorea & 765.9 & 0.5 & 93.2 & 24. Taiwan & 1'453.6 & 0.8 & 85.2 \\
\hline 25. Russland & 749.5 & 0.5 & 93.7 & 25. Tschechische Rep. & 1'441.5 & 0.8 & 86.0 \\
\hline 26. Thailand & 727.4 & 0.4 & 94.1 & 26. Dänemark & 1'315.8 & 0.7 & 86.7 \\
\hline 27. Türkei & 671.1 & 0.4 & 94.5 & 27. Griechenland & 1'295.4 & 0.7 & 87.5 \\
\hline 28. Indien & 637.6 & 0.4 & 94.9 & 28. Mexiko & 1'279.3 & 0.7 & 88.2 \\
\hline 29. Taiwan & 615.5 & 0.4 & 95.3 & 29. Saudi-Arabien & 1'215.0 & 0.7 & 88.9 \\
\hline 30. Portugal & 514.2 & 0.3 & 95.6 & 30. Ungarn & $1^{\prime} 006.2$ & 0.6 & 89.4 \\
\hline Übrige Länder & 7'330.9 & 4.4 & 100.0 & Übrige Länder & $18^{\prime} 723.9$ & 10.6 & 100.0 \\
\hline Gesamtbetrag & $165^{\prime} 410.3$ & & 100.0 & Gesamtbetrag & $177^{\prime} 474.8$ & & 100.0 \\
\hline
\end{tabular}

Quelle: Eidgenössische Zollverwaltung, Aussenhandel der Schweiz. Statistik nach Ländern, Jahr 2006, CD-ROM.

Anmerkung: Aussenhandel ohne Edelmetalle, Schmucksteine, Kunstgegenstände und Antiquitäten (Gesamtwert 1 der Zollstatistik). Der Goldhandel ist sogar aus der Zollstatistik ganz ausgeschlossen.

a Die Entwicklungsländer (gemäss DAC-Liste) sind fett gedruckt.

b Anteil des Landes an den Gesamteinfuhren aus den 30 wichtigsten Handelspartnern, bzw. an den Gesamtausfuhren in diese Partnerländer.

c Kumulierter Anteil der wichtigsten Länder an den Gesamteinfuhren aus den 30 wichtigsten Handelspartnern, bzw. an den Gesamtausfuhren in diese Partnerländer. 


\section{B.1.2 Aussenhandel der Schweiz mit den Entwicklungsländern und anderen Ländern des Südens und Ostens}

Das Schema B.1 zeigt die Beträge des Aussenhandels der Schweiz mit den Entwicklungsländern im Jahr 2006. Die Handelsströme sind nach Länderkategorien $^{3}$, gemäss der Länderklassifikation des DAC nach dem Einkommensstand pro Einwohner aufgeführt. Man stellt fest, dass die Ausfuhren in die Entwicklungsländer die Einfuhren aus diesen Ländern überschritten haben. Demnach verzeichnet die Schweiz einen Handelsüberschuss mit allen Kategorien von Ländern, einschliesslich der am wenigsten entwickelten Länder. Der grösste Teil des Aussenhandels der Schweiz mit den Entwicklungsländern entfällt aber auf Länder mit höherem Einkommen (Länder mit mittlerem Einkommen).

Der Handel mit den 50 am wenigsten entwickelten Ländern (LDC) ist gering. Die Ausfuhren in diese Länder machten 2006 mit 505,6 Millionen Franken lediglich 0,29 Prozent der Gesamtexporte der Schweiz aus. Mit Einfuhren in Höhe von 286 Millionen Franken im gleichen Jahr aus den am wenigsten entwickelten Ländern machten diese Länder nur 0,17 Prozent der Gesamtimporte der Schweiz aus. Ferner beliefen sich die Ausfuhren in die übrigen einkommensschwachen Länder (ohne LDC) für 2006 auf 2,7 Milliarden Franken und die Einfuhren aus diesen Ländern auf 2,1 Milliarden Franken. Somit erreichte der Handelsüberschuss mit dieser Kategorie von Ländern 611000 Franken und der Handelsüberschuss mit den Ländern mit mittlerem Einkommen belief sich auf 8,3 Milliarden Franken.

\section{Schema B.1: Übersicht über den Aussenhandel der Schweiz mit den Entwicklungsländern (EL), nach Einkommenskategorien der Länder, 2006 (in Millionen Franken)}

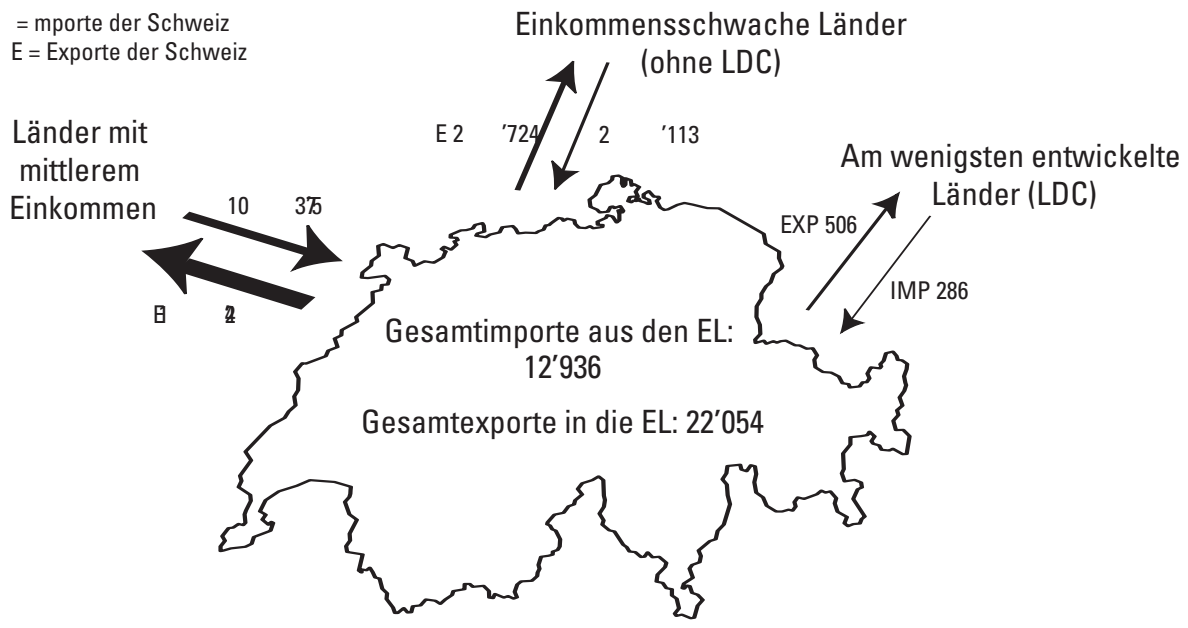

Quellen: Richard Gerster, Aus Fehlern lernen? Die Schweiz und die Dritte Welt, Zurich, Edition Ex Libris, 1987, S. 65. Oberzolldirektion, Schweizerische Aussenhandelsstatistik, Statistik nach Ländern. Jahr 2006, CD-ROM.

3 Ausführliche Angaben nach Ländern finden sich im Teil A der vorliegenden „Statistiken“. 


\section{$\square$ Handelsüberschüsse}

Seit mehreren Jahren weist die Handelsbilanz der Schweiz mit den Oststaaten, den Entwicklungsländern und den aufstrebenden Ländern insgesamt einen Überschuss aus, hingegen verzeichnet sie mit den Staaten der Europäischen Union ein bedeutendes Defizit (2006 in Höhe von über 24 Milliarden Franken). Anders gesagt, exportiert die Schweiz im Allgemeinen mehr in die aufstrebenden Länder, die Oststaaten oder die Entwicklungsländer, als sie aus diesen Ländern importiert. Folglich stellen diese Länder für die Schweizer Exporteure interessante Absatzmärkte dar.

Insgesamt erreichte der Handelsüberschuss mit den Entwicklungsländern im Jahr 2006 9,1 Milliarden Franken. Mit den Ländern Asiens, Afrikas und Lateinamerikas, die nicht mehr auf der Liste der Entwicklungsländer stehen - d.h. die fortgeschrittensten Volkswirtschaften - beträgt der Handelsüberschuss sogar über 10 Milliarden Franken.

Die Grafik B.2 führt diejenigen Länder unter den Entwicklungsländern und anderen Ländern des Südens- und Ostens auf, mit denen die Schweiz den grössten Handelsüberschuss verzeichnet. Der Überschuss mit Hongkong beträgt mehr als 2,7 Milliarden Franken. Mit der Türkei, Russland, Singapur, Indien, den Vereinten Arabischen Emiraten, Mexiko und Saudi-Arabien beträgt er über eine Millarde Franken. Es ist zu vermerken, dass die Schweiz einen bedeutenden Handelsüberschuss mit mehreren Ländern hat, die (besonders wegen der Risiken der Auslagerung von Arbeitsplätzen in diese Länder) oft als starke Konkurrenten für den Wirtschaftsstandort Schweiz und für die Beschäftigung in der Schweiz angesehen werden.

Grafik B.2: Entwicklungsländer (gemäss DAC-Klassifikation) und andere Länder des Südens und Ostens, mit denen der Handelsüberschuss der Schweiz am höchsten ist, 2006 (in Millionen Franken)

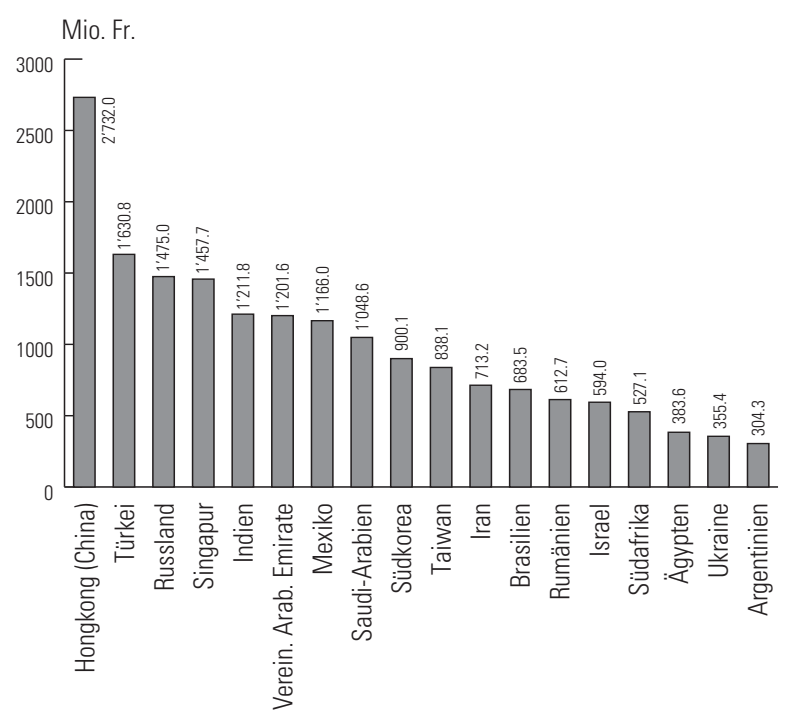

Quelle: Eidgenössische Zollverwaltung, http://www.ezv.admin.ch >Themen >Aussenhandelsstatistik >Länder $>$ Aussenhandel nach Wirtschaftsräumen und Kontinenten. 
Tabelle B.2: Die 30 wichtigsten Handelspartner der Schweiz unter den Entwicklungsländerna 2006 (in Millionen Franken, Anteile und kumulierte Anteile in Prozent)

\begin{tabular}{|c|c|c|c|c|c|c|c|}
\hline \multicolumn{4}{|c|}{ Wichtigste Bezugsländer } & \multicolumn{4}{|c|}{ Wichtigste Absatzländer } \\
\hline \multirow[t]{2}{*}{ Land $^{a}$} & \multirow[b]{2}{*}{ Mio. Fr. } & \multicolumn{2}{|c|}{ Einfuhren } & \multirow[t]{2}{*}{ Land $^{\mathrm{a}}$} & \multicolumn{3}{|c|}{ Ausfuhren } \\
\hline & & $\%^{b}$ & $\sum \%^{\mathrm{c}}$ & & Mio. Fr. & $\%^{\mathrm{b}}$ & $\sum \%$ \\
\hline 1. China & $3^{\prime} 918.6$ & 30.3 & 30.3 & 1. China & $3{ }^{\prime} 753.0$ & 17.0 & 17.0 \\
\hline 2. Libyen & 1'677.2 & 13.0 & 43.3 & 2. Türkei & $2 \prime 301.9$ & 10.4 & 27.5 \\
\hline 3. Nigeria & $1 ' 046.5$ & 8.1 & 51.3 & 3. Indien & 1'849.4 & 8.4 & 35.8 \\
\hline 4. Brasilien & 775.0 & 6.0 & 57.3 & 4. Brasilien & 1'458.5 & 6.6 & 42.5 \\
\hline 5. Thailand & 727.4 & 5.6 & 63.0 & 5. Mexiko & 1'279.3 & 5.8 & 48.3 \\
\hline 6. Türkei & 671.1 & 5.2 & 68.2 & 6. Saudi-Arabien & 1'215.0 & 5.5 & 53.8 \\
\hline 7. Indien & 637.6 & 4.9 & 73.1 & 7. Thailand & 939.9 & 4.3 & 58.0 \\
\hline 8. Kasachstan & 419.6 & 3.2 & 76.3 & 8. Iran & 747.7 & 3.4 & 61.4 \\
\hline 9. Malaysia & 245.3 & 1.9 & 78.2 & 9. Südafrika & 727.4 & 3.3 & 64.7 \\
\hline 10. Vietnam & 224.2 & 1.7 & 80.0 & 10. Malaysia & 513.7 & 2.3 & 67.0 \\
\hline 11. Südafrika & 200.3 & 1.5 & 81.5 & 11. Ukraine & 423.3 & 1.9 & 69.0 \\
\hline 12. Marokko & 169.0 & 1.3 & 82.8 & 12. Ägypten & 416.4 & 1.9 & 70.9 \\
\hline 13. Saudi-Arabien & 166.4 & 1.3 & 84.1 & 13. Argentinien & 361.9 & 1.6 & 72.5 \\
\hline 14. Indonesien & 150.2 & 1.2 & 85.3 & 14. Pakistan & 327.4 & 1.5 & 74.0 \\
\hline 15. Libanon & 140.4 & 1.1 & 86.3 & 15. Indonesien & 325.9 & 1.5 & 75.5 \\
\hline 16. Algerien & 128.2 & 1.0 & 87.3 & 16. Marokko & 280.2 & 1.3 & 76.7 \\
\hline 17. Mexiko & 113.2 & 0.9 & 88.2 & 17. Kroatien & 271.2 & 1.2 & 78.0 \\
\hline 18. Bangladesch & 108.2 & 0.8 & 89.0 & 18. Libanon & 253.8 & 1.2 & 79.1 \\
\hline 19. Philippinen & 76.8 & 0.6 & 89.6 & 19. Algerien & 248.0 & 1.1 & 80.2 \\
\hline 20. Ecuador & 75.7 & 0.6 & 90.2 & 20. Libyen & 239.4 & 1.1 & 81.3 \\
\hline 21. Kolumbien & 72.9 & 0.6 & 90.8 & 21. Venezuela & 233.6 & 1.1 & 82.4 \\
\hline 22. Costa Rica & 68.5 & 0.5 & 91.3 & 22. Kolumbien & 229.4 & 1.0 & 83.4 \\
\hline 23. Ukraine & 67.9 & 0.5 & 91.8 & 23. Kasachstan & 217.5 & 1.0 & 84.4 \\
\hline 24. Kroatien & 66.7 & 0.5 & 92.4 & 24. Philippinen & 216.9 & 1.0 & 85.4 \\
\hline 25. Pakistan & 65.9 & 0.5 & 92.9 & 25. Panama & 201.4 & 0.9 & 86.3 \\
\hline 26. Chile & 62.5 & 0.5 & 93.3 & 26. Chile & 196.2 & 0.9 & 87.2 \\
\hline 27. Argentinien & 57.6 & 0.4 & 93.8 & 27. Nigeria & 191.1 & 0.9 & 88.1 \\
\hline 28. Ghana & 50.5 & 0.4 & 94.2 & 28. Serbien & 187.3 & 0.8 & 88.9 \\
\hline 29. Sri Lanka & 50.5 & 0.4 & 94.6 & 29. Jordanien & 183.3 & 0.8 & 89.7 \\
\hline 30. Aserbaidschan & 43.5 & 0.3 & 94.9 & 30. Vietnam & 140.4 & 0.6 & 90.4 \\
\hline $\begin{array}{l}\text { Übrige } \\
\text { Entwicklungsländer }\end{array}$ & 658.6 & 5.1 & 100.0 & $\begin{array}{l}\text { Übrige } \\
\text { Entwicklungsländer }\end{array}$ & $2^{\prime} 123.0$ & 9.6 & 100.0 \\
\hline Gesamtbetrag & $12^{\prime} 935.8$ & & 100.0 & Gesamtbetrag & $22 ' 053.5$ & & 100.0 \\
\hline
\end{tabular}

Quelle: Eidgenössische Zollverwaltung, Aussenhandel der Schweiz. Statistik nach Ländern, Jahr 2006, CD-R0M. Anmerkung: Aussenhandel ohne Edelmetalle, Schmucksteine, Kunstgegenstände und Antiquitäten (Gesamtwert 1 der Zollstatistik). Der Goldhandel ist sogar aus der Zollstatistik ganz ausgeschlossen.

a Entwicklungsländer (gemäss DAC-Liste).

b Anteil des Landes an den Gesamteinfuhren der Schweiz aus den Entwicklungsländern, bzw. an den Gesamtausfuhren in diese Länder.

c Kumulierter Anteil der wichtigsten Länder an den Gesamteinfuhren aus den Entwicklungsländern, bzw. an den Gesamtausfuhren in diese Länder.

Zusätzliche Tabellen und Grafiken sind auf der Website des Schweizerischen Jahrbuchs für Entwicklungspolitik unter <http://graduateinstitute.ch/development/publications/aspd.html> zu finden.

Tabelle B.2 führt die 30 wichtigsten Handelspartner der Schweiz unter den Entwicklungsländern auf. 2006 hat die Schweiz Waren in Höhe von insgesamt 22,1 Milliarden Franken in die Entwickungsländer exportiert und Produkte für insgesamt 12,9 Milliarden Franken aus diesen Ländern importiert. Somit belief sich der Handelsüberschuss mit dieser Ländergruppe auf 9,1 Milliarden Franken. Ein Teil 
des Wohlstands der Schweiz hängt demzufolge von den Ausfuhren in die Entwicklungsländer $a b$. Der relativ geringe Umfang der Einfuhren aus einigen Entwicklungsländern zeigt das Potenzial zur Verbesserung des Zugangs bestimmter Agrar- und Industrieprodukte zum Schweizer Markt für die Entwicklung dieser Partnerländer auf. Eine Senkung der Zollschranken und der nichttarifären Hindernisse für die Einfuhren aus den Entwicklungsländern könnte den Marktzugang verbessern. Auch sind Initiativen wie das Schweizerische Importförderungsprogramm (SIPPO) bestrebt, den Entwicklungsländern zu einer besseren Nutzung ihrer Exportmöglichkeiten nach der Schweiz zu verhelfen.

\section{B.2. Direktinvestitionen im Ausland und Bankanlagen}

\section{B.2.1. Direktinvestitionen im Ausland}

Durch ihre Stabilität und ihre Zielausrichtung fördern die Direktinvestitionen im Ausland den Technologietransfer und die Schaffung von Arbeitsplätzen und unterscheiden sich dadurch von den volatileren Portfolioanlagen. Aus der Sicht der Entwicklung sind diese Investitionen in den Empfängerländern öffentlicher Entwicklungshilfe von gewisser Bedeutung, denn „mit Direktinvestitionen soll ein dauerhafter und direkter Einfluss auf die Geschäftstätigkeit eines Unternehmens im Ausland ausgeübt werden" .

\section{$\square$ Direktinvestitionsströme im Ausland, 2006}

Im Jahr 2006 erreichten die schweizerischen Direktinvestitionen im Ausland den Rekordbetrag von 87,6 Milliarden Franken (2005 : 64 Milliarden Franken) ${ }^{5}$. Das letzte Rekordhoch vom Jahr 2000 hatte 75 Milliarden Franken erreicht. Die beträchtliche Zunahme der Direktinvestitionen von 2005 auf 2006 erklärt sich vor allem durch das starke Weltwirtschaftswachstum und die hohen Liquiditäten der Unternehmen.

Der grösste Teil der Investitionen ging in die Staaten der Europäischen Union (28,6 Milliarden Franken) und nach Nordamerika (23,3 Milliarden Franken). Die Investitionen in die Entwicklungsländer werden auf 12,5 Milliarden Franken geschätzt, davon flossen 37 Millionen Franken in die am wenigsten entwickelten Länder ${ }^{6}$. Die Direktinvestitionsströme nach Afrika betrugen 797 Millionen Franken, davon flossen 427 Millionen Franken nach Südafrika und 160

4 Schweizerische Nationalbank (SNB), Die Entwicklung der Direktinvestitionen 2006, Zürich, SNB, Dezember 2007, S. 15.

5 Quelle: Ibid., Tabelle 1.1, „Schweizerische Direktinvestitionen im Ausland. Ländergliederung der Kapitalexporte“, S. A2-A3. Hinweis: Die in Teil A der vorliegenden „Statistiken“ veröffentlichten Länderdaten (Quelle: Statistischer Dienst der DEZA anhand der fragmentarischen Angaben der SNB zu einigen Ländern) entsprechen nicht den aktualisierten und vollständigeren Daten, die in der Publikation der Schweizerischen Nationalbank im Dezember 2007 erscheinen.

6 Bei einer Analyse der Statistiken der SNB über die Investitionen ist ein Hinweis erforderlich. Die Länderklassifikation der SNB unterscheidet sich stark von der DAC-Klassifikation: In den Statistiken der SNB können die Entwicklungsländer der DAC-Liste im restlichen Europa eingeordnet sein (Türkei und Ukraine). Ferner enthalten die Einstufungen der SNB nach Kontinenten für Lateinamerika, Asien und Afrika Länder der DAC-Liste der Entwicklungsländer, aber auch andere fortgeschrittenere Länder, die nicht auf der DAC-Liste stehen, wie gewisse Offshore-Finanzzentren oder Industrieländer Übersee (Japan). Betreffend die Direktinvestitionen im Ausland gemäss der DAC-Liste wird auf die Tabelle im Teil A der vorliegenden ,Statistiken“ verwiesen. 
Millionen Franken nach Ägypten7 . Die Investitionen in den aussereuropäischen Offshore-Finanzzentren ${ }^{8}$ beliefen sich 2006 auf 16,2 Milliarden Franken und die Investitionen in Singapur auf 4,4 Milliarden Franken. Die in diesen Ländern betroffenen Tochtergesellschaften dienen oft als Drehscheibe für Investitionen in Drittländern (Holdingfunktion).

\section{$\square$ Bestand der Direktinvestitionen im Ausland Ende 2006}

Die Grafik B.3 zeigt die regionale Verteilung des Bestands der schweizerischen Direktinvestitionen im Ausland Ende 2006 auf. Der Bestand belief sich auf über 632 Milliarden Franken, wovon mehr als 41 Prozent auf die Europäische Union entfielen. 23,7 Prozent gingen nach Nordamerika und rund 20 Prozent nach Zentral- und Südamerika. Dahinter folgen Asien mit 8,4 Prozent der Direktinvestitionen im Ausland, die übrigen europäischen Länder mit rund 5,8 Prozent und Ozeanien mit 1,6 Prozent. Der Bestand der schweizerischen Direktinvestitionen in Afrika belief sich auf 7,5 Milliarden Franken (1,2\% des Gesamtbestands), wovon rund 5 Milliarden Franken auf Südafrika, 510 Millionen auf Ägypten und 337 Millionen auf Marokko entfielen'.

\section{Grafik B.3: Aufteilung der schweizerischen Direktinvestitionsbestände nach Regionen, per 31. Dezember 2006 (in Millionen Franken und Prozent)}

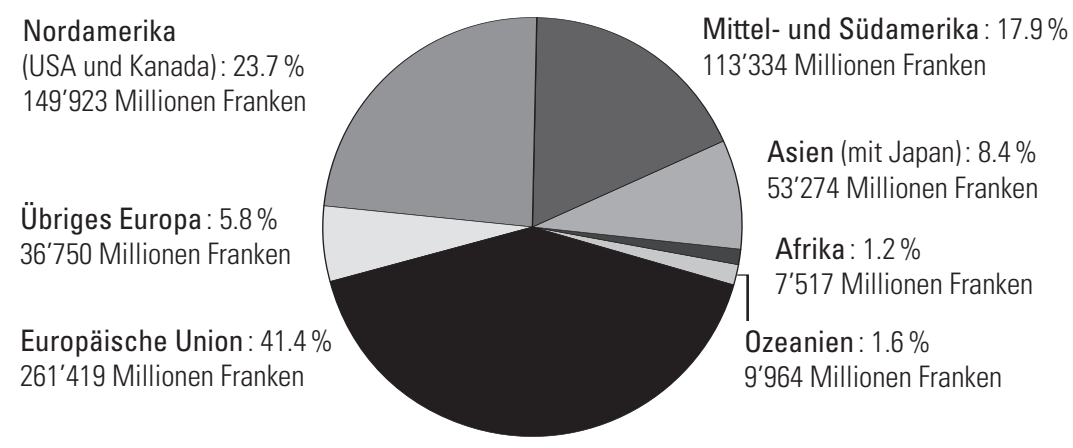

Welt insgesamt: 632'181 Millionen Franken

Quelle: Schweizerische Nationalbank, Die Entwicklung der Direktinvestitionen 2006, Zürich, SNB, Dezember 2007, Tabelle 1.2, „Schweizerische Direktinvestitionen im Ausland. Ländergliederung des Kapitalbestandes (Jahresende)”, S. A4-A5.

\section{$\square$ Entwicklung des Investisionsbestands für ausgewählte Länder}

Die Grafiken B.4 und B.5 beziehen sich auf die wichtigsten Direktinvestitionsbestände der Schweiz in den Empfängerländern öffentlicher Entwickungshilfe und in einer Reihe anderer ausgewählter Länder des Südens und Ostens, in einem

7 Quelle: SNB, op. cit., Tabelle 1.1, „Schweizerische Direktinvestitionen im Ausland. Ländergliederung der Kapitalexporte", S. A2-A3.

8 Amerikanische Jungferninseln, Anguilla, Antigua und Barbuda, Bahamas, Barbados, Belize, Bermuda-Inseln, Britische Jungferninseln, Dominica, Grenada, Jamaika, Cayman-Inseln, Montserrat, Niederländische Antillen, Panama, St. Kitts und Nevis, St. Lucia, St. Vincent und Grenadinen, Turksund Caicosinseln (in Kursivschrift: Länder, die nicht auf der DAC-Liste der Empfängerländer öffentlicher Entwicklungshilfe aufgeführt sind).

9 Quelle: SNB, op. cit., Tabelle 1.2, „Schweizerische Direktinvestitionen im Ausland. Ländergliederung des Kapitalbestandes (Jahresende)“, S. A4-A5. 
Abstand von vier Jahren (Ende 2002 und Ende 2006). Dabei fällt sofort der bedeutende Umfang der Direktinvestitionen im Ausland in den Offshore-Finanzzentren ${ }^{10}$ der Karibik sowohl Ende 2002 als auch Ende 2006, mit einer Zunahme um 44,8 Milliarden Franken zwischen diesen beiden Daten auf (Grafik B.5).

Der Gesamtbestand der Investitionen in den Entwickungsländern der Grafik B.4 beläuft sich 2006 auf 43,5 Milliarden Franken (bei einem Gesamtbestand an Auslandsinvestitionen von 632 Milliarden Franken). Dazu ist zu vermerken, dass die Mehrheit der Entwicklungsländer von den Direktinvestitionen der Schweiz ausgeschlossen bleibt. In der Tat führen die Statistiken der SNB lediglich 17 Entwicklungsländer mit einem Investitionsbestand von über 500 Millionen Franken auf. Ende 2006 waren die Entwicklungsländer mit den höchsten Schweizer Investitionsbeständen Brasilien (mit 10,1 Milliarden Franken), Südafrika (rund 5 Milliarden Franken) und Mexiko (4,8 Miliarden Franken).

Was die Entwicklung der Beträge zwischen Ende 2002 und Ende 2006 betrifft, so hat sich der Bestand der Schweizer Investitionen in der Ukraine versiebenfacht, in Südafrika und Indonesien vervierfacht, in Indien und Pakistan mehr als verdreifacht und in Brasilien, Chile, China sowie in der Türkei mehr als verdoppelt. Andere Länder verzeichneten hingegen einen Rückgang des Direktinvestitionsbestands der Schweiz zwischen Ende 2002 und Ende 2006. Dies war namentlich bei Kolumbien (mit einem Rückgang um 16 Prozent) der Fall.

\section{Grafiken B.4 und B.5: Wichtigste Bestimmungsländer und -regionen der schweizerischen Direktinvestitionen, Bestand Ende 2002 und Ende 2006 (in Mio. F.)}

\section{Grafik B.4: Empfängerländer öffentlicher Entwicklungshilfe gemäss DAC-Liste}

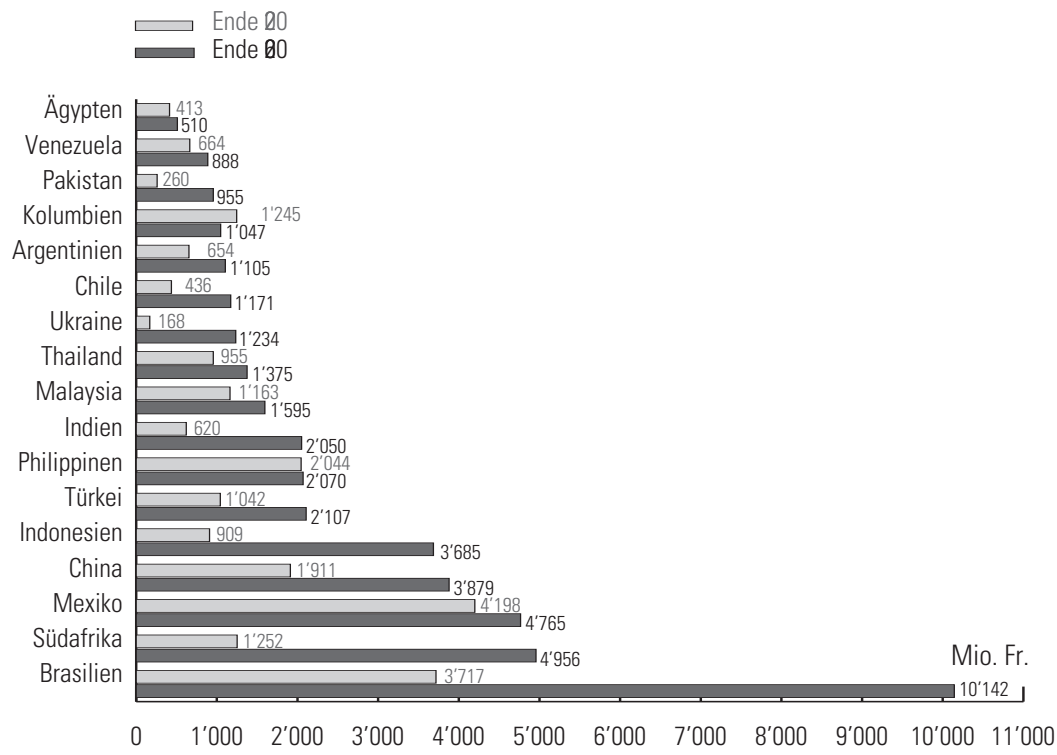

10 Unter den Ländern oder Gebieten des Aggregats der Offshore-Finanzzentren der Schweizerischen Nationalbank sind einige für die Beträge ab 2005 nicht (oder nicht mehr) auf der DAC-Liste der Empfängerländer öffentlicher Entwicklungshilfe aufgeführt. Dabei handetl es sich um die Amerikanischen Jungferninseln, die Bahamas, die Bermuda-Inseln, die Britischen Jungferninseln, die CaymanInseln und die Niederländischen Antillen. 


\section{Grafik B.5: Andere Länder des Südens und Ostens}

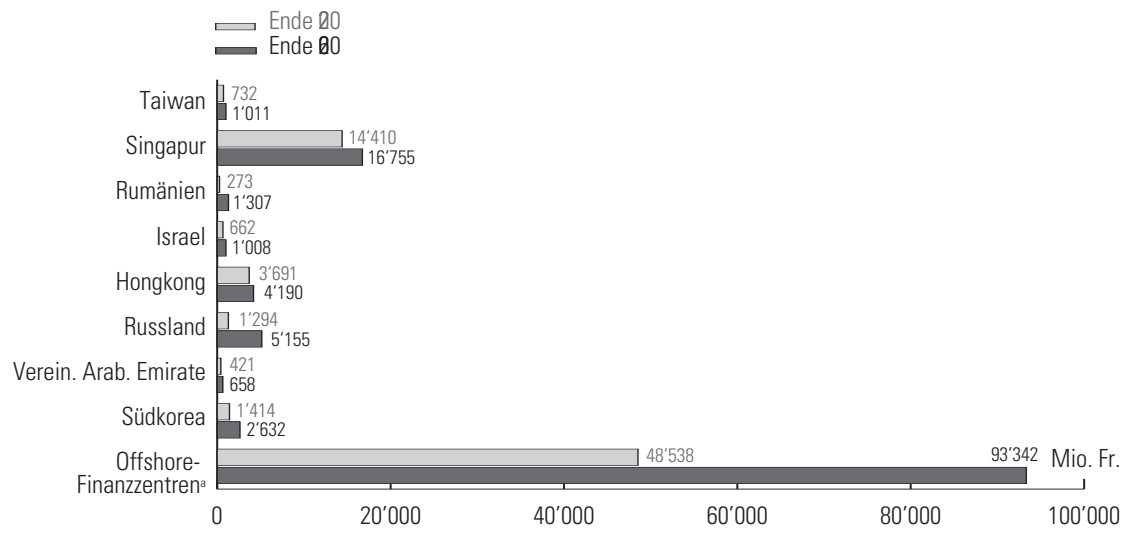

Gemäss der Klassifikation der SNB umfassen die Offshore-Finanzzentren Entwicklungsländer und andere Länder, die nicht auf der DAC-Liste der Empfängerländer öffentlicher Entwickungshilfe stehen: Amerikanische Jungferninseln, Anguilla, Antigua und Barbuda, Bahamas, Barbados, Belize, Bermuda-Inseln, Britische Jungferninseln, Dominica, Grenada, Jamaika, Cayman-Inseln, Montserrat, Niederländische Antillen, Panama, St. Kitts und Nevis, St. Lucia, St. Vincent und Grenadinen, Turks- und Caicosinseln.

Quelle der Grafiken B.4 und B.5: Schweizerische Nationalbank, Entwicklung der Direktinvestitionen 2006, Zürich, SNB, Dezember 2007, Tabelle 1.2, „Schweizerische Direktinvestitionen im Ausland. Ländergliederung des Kapitalbestandes (Jahresende)", S. A4-A5.

\section{B.2.2 Anlagen in Schweizer Banken}

Der Finanzplatz Schweiz ist von grosser wirtschaftlicher Bedeutung. Der Bankensektor beschäftigt rund 128000 Angestellte ${ }^{11}$. Gemäss Schätzungen des Eidgenössischen Finanzdepartements belaufen sich die vom Finanzsektor (Banken und Versicherungen) gezahlten Einkommens- und Unternehmenssteuern auf mindestens 10 Prozent der Steuereinnahmen in der Schweiz ${ }^{12}$. Laut der Stiftung Genève Place financière, die sich auf Statistiken der Schweizerischen Nationalbank stützt ${ }^{13}$, wurde der Gesamtbetrag der Kundeneinlagen in den Schweizer Bankinstituten im November 2007 auf 5341 Milliarden Franken geschätzt (4577 Milliarden Franken im Mai 2006); davon entfielen 59,6 Prozent auf ausländische Kunden (institutionelle Kunden: 35,5\%, Unternehmen: 4,3\%, Privatkunden: 19,8\%).

Die Statistiken der Schweizerischen Nationalbank unterscheiden die von den Banken auf eigene Rechnung und Gefahr getätigten (in der Bilanz aufgeführten) Geschäfte von den auf Rechnung und Gefahr ihrer Kunden getätigten Transaktionen (Treuhandgeschäfte). Die Angaben zum Finanzplatz Schweiz sind nicht vollständig, da die Publikationen der SNB in diesem Bereich lediglich die Zahlen für rund 100 Banken in der Schweiz (2006: 95 Banken) berücksichtigen und die Beträge des Parabankenbereichs sowie die von privaten Vermögensverwaltern betreuten Gelder de facto ausschliessen.

13 Genève Place financière Grafik „Stocks de titres dans les dépôts de la clientèle auprès des banques en Suisse“, http://www.geneve-finance.ch/fr/place_fin/place_fin_ch/faits_chiffres_ch> (Einsichtnahme: 8 . April 2008). SNB, Statistisches Monatsheft, Zürich, November 2007. 
Gemäss den von der SNB veröffentlichten Zahlen sind die Depots aus Entwicklungsländern, aufstrebenden Ländern oder Offshore-Finanzplätzen bei den Banken in der Schweiz im Allgemeinen wesentlich höher als die Guthaben der Schweizer Banken in diesen Ländern. Mit anderen Worten nimmt der Finanzplatz Schweiz viel mehr Kapital aus afrikanischen, asiatischen und lateinamerikanischen Ländern auf, als er diesen Ländern bereitstellt.

\section{Grafik B.6: Netto-Treuhandverpflichtungen der in der Schweiz niedergelassenen Banken, Gelder aus den Entwicklungsländern, per 31. Dezember 2006 (in Mio. Franken)}

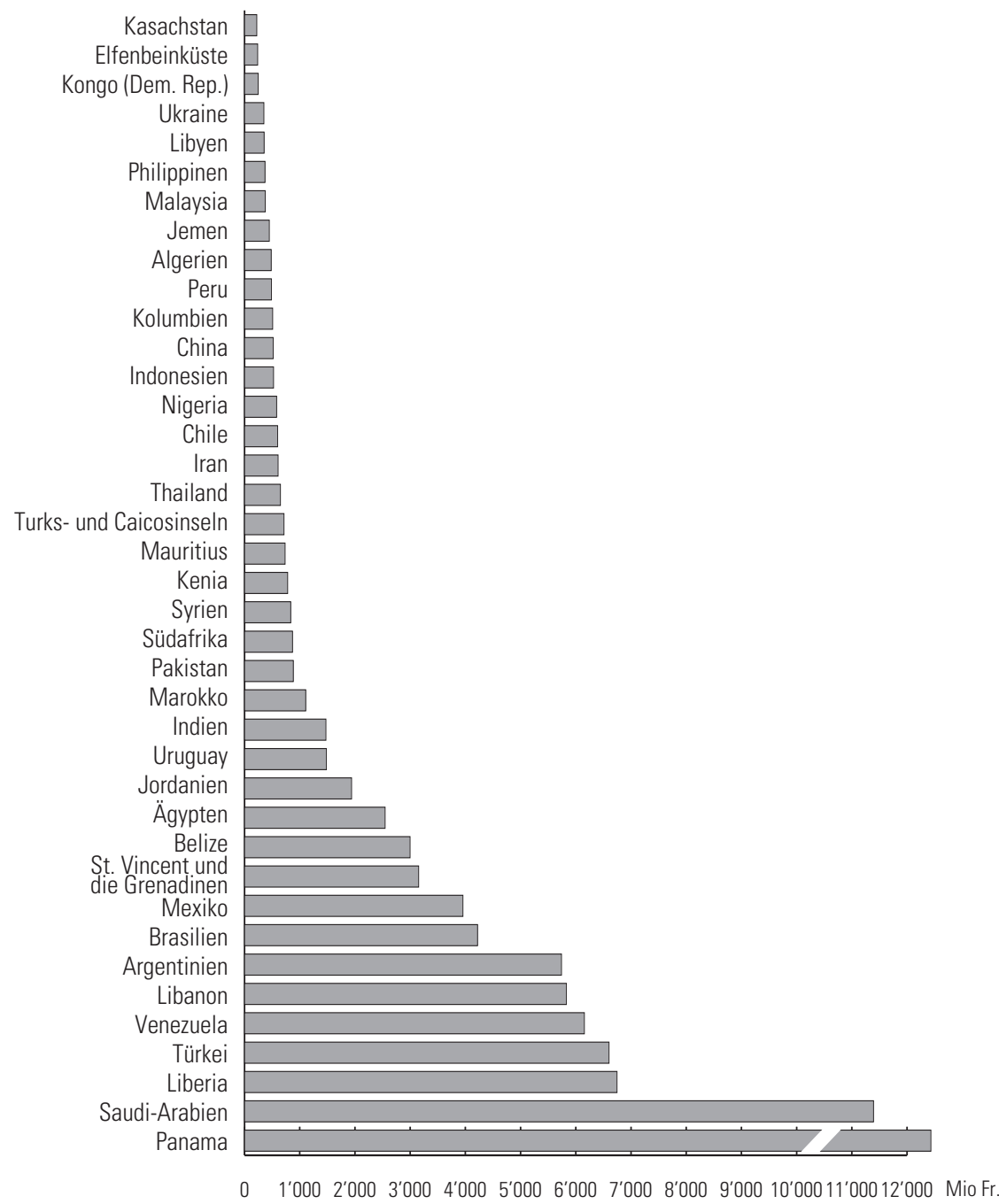

Quelle: Schweizerische Nationalbank, Die Banken in der Schweiz 2006, Zürich, SNB, Juni 2007, Tabelle 38, „Treuhandgeschäfte - Länderweise Gliederung", S. A132-A137. 
Die Grafik B.6 zeigt die Netto-Treuhandanlagen in den von der Statistik der Schweizerischen Nationalbank erfassten 95 Banken aus den Empfängerländern öffentlicher Entwicklungshilfe per Ende 2006 auf. Unter diesen Ländern befinden sich eine Reihe von Offshore-Finanzzentren, darunter an erster Stelle Panama mit rund 32,4 Milliarden Franken an Treuhandanlagen in der Schweiz. Auch sind dort Länder aufgeführt, die indessen auf der Liste der am wenigsten entwickelten Länder stehen, darunter Liberia (6,7 Milliarden Franken), der Jemen (448 Millionen Franken), die Demokratische Republik Kongo (247 Millionen Franken) und Angola (101 Millionen Franken). Ferner findet man auf der Liste sonstige einkommensschwache Länder wie Pakistan (882 Millionen Franken), Kenia (780 Millionen), Nigeria (560 Millionen) und Kasachstan (221 Millionen Franken). Weniger erstaunlich sind dagegen die Netto-Verpflichtungen im Treuhandgeschäft von rohstoffreichen (insbesondere Erdöl produzierenden) Ländern wie beispielsweise Saudi-Arabien (11,4 Milliarden Franken).

Zusätzliche Daten, Tabellen und Kommentare zu den Finanzflüssen in die Enwicklungsländer, den Direktinvestitionen im Ausland und den Bankanlagen finden sich auf der Internetseite des Schweizerischen Jahrbuchs für Entwicklungspolitik unter: <http://graduateinstitute.ch/development/publications/aspd.html>.

\section{B.3. Öffentliche Entwicklungshilfe}

\section{B.3.1. Öffentliche Entwicklungshilfe der Mitgliedsländer des OECD-Entwicklungshilfeausschusses (DAC)}

Tabelle B.3 gibt eine Übersicht über die öffentliche Entwicklungshilfe der Mitgliedsländer des OECD-Entwicklungshilfeausschusses (DAC). Sie zeigt einen Rückgang der öffentlichen Entwicklungshilfe von 2005 auf 2006 an. Die Gesamtleistungen an öffentlicher Hilfe sind von 107,1 Milliarden Dollar im Jahr 2005 auf 104,4 Milliarden Dollar im Jahr 2006 gesunken. Dieser Rückgang der Hilfe um 4,5 Prozent erklärt sich weitgehend durch geringere Beträge für die Schuldenerleichterungen, die 2005 Rekordhöhen erreicht hatten. Aber auch ohne diese Schuldenerleichterungen ist die öffentliche Entwicklungshilfe real um 0,8 Prozent gesunken, trotz der Verpflichtungen seitens der G-8 im Jahr 2005 und des Millennium+5-Gipfels, die Entwicklungshilfe von 2004 bis 2010 real um 50 Milliarden zu erhöhen und die Hilfe für Afrika zu verdoppeln.

Die öffentliche Entwicklungshilfe (APD) setzt sich gemäss Definition der OECD aus allen Finanzflüssen zusammen, die

$\square$ aus dem öffentlichen Sektor stammen (in der Schweiz: Bund, Kantone und Gemeinden);

$\square$ vorrangig auf die Erleichterung der wirtschaftlichen und sozialen Entwicklung der Empfängerländer abzielen ;

$\square \mathrm{zu}$ Vorzugsbedingungen gewährt werden (Schenkungen oder Darlehen zu günstigen Konditionen);

๖ für Entwicklungsländer oder -gebiete bestimmt sind sowie für multilaterale Organisationen, die auf der OECD-Liste stehen. Seit 2006 umfasst die DACListe die Länder, deren Volkseinkommen pro Einwohner im Jahr 2004 unter 10065 Dollar lag. Das DAC berechnet nicht mehr das getrennte Aggregat für 
öffentliche Hilfe, das von 1993 bis 2004 die Transitionsländer (osteuropäische Staaten und sog. fortgeschrittenere Entwicklungsländer) betroffen hatte.

Die von den DAC-Ländern aufgebrachte öffentliche Entwicklungshilfe belief sich 2006, anteilmässig am Bruttonationaleinkommen (BNE), auf 0,46 Prozent (2005: 0,47\%). Nur folgende fünf Länder haben das von den Vereinten Nationen festgesetzte Ziel von 0,7 Prozent des BNE erreicht oder überschritten: Dänemark, Luxemburg, Niederlande, Norwegen und Schweden.

Tabelle B.3: Öffentliche Entwicklungshilfe (APD) der DAC-Länder (nach Ländern) und Veränderung von 2005 auf 2006 (in Millionen Dollar und in Prozent des BNE)

\begin{tabular}{|c|c|c|c|c|c|c|}
\hline \multirow[t]{2}{*}{ Land } & \multicolumn{2}{|c|}{$\begin{array}{c}\text { APD (in Millionen } \\
\text { Dollar) }\end{array}$} & \multicolumn{2}{|c|}{$\begin{array}{l}\text { APD (in \% } \\
\text { des BNE) }\end{array}$} & \multirow{2}{*}{$\begin{array}{c}\text { Reale } \\
\text { Veränderung }^{\mathrm{b}} \\
2005 \text { bis } 2006 \\
\end{array}$} & \multirow{2}{*}{$\begin{array}{l}\text { Reale Veränderung } \\
\text { ohne Schuldener- } \\
\text { leichterungen }\end{array}$} \\
\hline & 2005 & 2006 & 2005 & 2006 & & \\
\hline Deutschland & $10^{\prime} 082$ & $10^{\prime} 435$ & 0.36 & 0.36 & 1.7 & 14.2 \\
\hline Australien & 1'680 & $2^{\prime} 123$ & 0.25 & 0.30 & 22.5 & 7.8 \\
\hline Österreich & $1^{\prime} 573$ & 1'498 & 0.52 & 0.47 & -6.9 & 8.3 \\
\hline Belgien & $1^{\prime} 963$ & $1^{\prime \prime} 978$ & 0.53 & 0.50 & -2.2 & 2.9 \\
\hline Kanada & $3^{\prime} 756$ & $3^{\prime} 684$ & 0.34 & 0.29 & -9.9 & -4.7 \\
\hline Dänemark & $2^{\prime} 109$ & $2 ' 236$ & 0.81 & 0.80 & 3.0 & -0.8 \\
\hline Spanien & $3^{\prime} 018$ & $3^{\prime} 814$ & 0.27 & 0.32 & 20.7 & 31.5 \\
\hline USA & 27 '935 & $23^{\prime} 532$ & 0.23 & 0.18 & -18.2 & -10.6 \\
\hline Finnland & 902 & 834 & 0.46 & 0.40 & -9.0 & 9.2 \\
\hline Frankreich & $10^{\prime} 026$ & $10^{\prime} 601$ & 0.47 & 0.47 & 2.9 & 2.3 \\
\hline Griechenland & 384 & 424 & 0.17 & 0.17 & 5.9 & 5.9 \\
\hline Irland & 719 & $1^{\prime} 022$ & 0.42 & 0.54 & 36.9 & 37.0 \\
\hline Italien & $5^{\prime} 091$ & $3^{\prime} 641$ & 0.39 & 0.20 & -30.6 & -42.0 \\
\hline Japan & $13^{\prime} 147$ & 11'187 & 0.28 & 0.25 & -9.1 & -8.9 \\
\hline Luxemburg & 256 & 291 & 0.82 & 0.89 & 4.8 & 4.8 \\
\hline Norwegen & 2'786 & 2'954 & 0.94 & 0.89 & -1.9 & -2.6 \\
\hline Neuseeland & 274 & 259 & 0.27 & 0.27 & 0.5 & 0.6 \\
\hline Niederlande & 5'115 & $5 ' 452$ & 0.82 & 0.81 & 4.2 & 4.9 \\
\hline Portugal & 377 & 396 & 0.21 & 0.21 & 2.0 & 2.8 \\
\hline Grossbritannien & $10^{\prime} 772$ & $12^{\prime} 459$ & 0.47 & 0.51 & 11.7 & 19.5 \\
\hline Schweden & $3^{\prime} 362$ & $3 \prime 955$ & 0.94 & 1.02 & 14.7 & 7.9 \\
\hline Schweiz & $1^{\prime} 772$ & 1'646 & 0.44 & 0.39 & -7.4 & -0.3 \\
\hline Total DAC & $107 ' 099$ & $104^{\prime} 421$ & 0.33 & 0.31 & -4.5 & -0.8 \\
\hline $\begin{array}{l}\text { Durchschnittliche Leistung } \\
\text { der DAC-Länder }\end{array}$ & & & 0.47 & 0.46 & & \\
\hline EU-Länder & $55^{\prime} 750$ & $59^{\prime} 035$ & 0.44 & 0.43 & 2.9 & 6.7 \\
\hline Mitgliedsländer der G-7 & $80^{\prime} 809$ & $75^{\prime} 539$ & 0.30 & 0.27 & -8.0 & -4.1 \\
\hline $\begin{array}{l}\text { Nichtmitgliedsländer } \\
\text { der G-7 }\end{array}$ & $26^{\prime} 290$ & $28^{\prime} 882$ & 0.50 & 0.51 & 6.3 & 7.5 \\
\hline $\begin{array}{l}\text { Nichtmitgliedsländer } \\
\text { des DACc }\end{array}$ & 3'905 & $5^{\prime} 172$ & & & & \\
\hline
\end{tabular}

Quelle:CAD, Statistical Annex of the Development Co-operation Report, Tabelle 1, Dezember 2007.

a Die Länder, die das von den Vereinten Nationen festgelegte Ziel von 0,7 \% des BNE übertreffen, sind fett gedruckt.

b Reale Veränderung der öffentlichen Entwicklungshilfe, inflations- und wechselkursbereinigt.

c Das DAC erfasst auch die Hilfeleistungen der OECD-Länder, die nicht Mitglied des DAC sind (Island, Korea, Polen, Slowakische Republik, Tschechische Republik, Türkei, Ungarn) sowie weiterer Geberländer (Chinesisch Taipeh, Israel, Thailand und andere). Tabelle 33 des Statistical Annex of the Development Co-operation Report führt länderweise partielle Daten zur öffentlichen Entwicklungshilfe der Nichtmitgliedsländer des DAC auf. Diese Daten sind jedoch unvollständig, da mehrere Nichtmitglieder der OECD keine Zahlenangaben zu ihren Hilfebeträgen machen. 


\section{B.3.2. Volumen der öffentlichen Entwicklungshilfe der Schweiz}

Vergleichbar mit der bei sämtlichen DAC-Ländern zu beobachtenden Tendenz ist die öffentliche Entwicklungshilfe der Schweiz von 2005 auf 2006 um 6,5 Prozent zurückgegangen, wobei sie von 2207,2 Millionen Franken auf 2063 Millionen Franken absank. Dieser Rückgang ist hauptsächlich durch den geringeren Betrag für Schuldenerleichterungen bedingt. Anteilmässig am BNE ist die öffentliche Entwicklungshilfe der Schweiz von 2005 auf 2006 von 0,44 Prozent auf 0,39 Prozent gesunken (siehe Grafik B.8). Hierzu sei vermerkt, dass die Hilfe der Schweiz bis 1990 regelmässig angestiegen war, sich dann in den 90er Jahren bei 0,34 Prozent stabilisierte und zwischen 2002 und 2005 zunahm. Die öffentliche Entwicklungshilfe macht im Jahr 2006 nur 1,46 Prozent der gesamten öffentlichen Ausgaben aus.

\section{$\square$ Jüngste Entwicklung der öffentlichen Hilfe der Schweiz}

Die Grafik B.7 zeigt, dass ein Grossteil der in letzter Zeit erfolgten Erhöhung der öffentlichen Entwicklungshilfe der Schweiz auf der Verbuchung neuer Elemente im Aggregat der öffentlichen Entwicklungshilfe beruht.

Die Zunahme der ,traditionellen“ bilateralen und multilateralen Hilfe ist zwar reell, doch fällt sie, verglichen mit den Elementen, die dem Entwicklungshilfeaggregat nach und nach hinzugefügt wurden, ziemlich gering aus. Von 2002 bis 2006 hat sich die von der Schweiz aufgebrachte öffentliche Entwicklungshilfe um 41,4 Prozent erhöht; ohne Berücksichtigung der dem statistischen Entwicklungshilfeaggregat hinzugefügten Elemente hat sie jedoch lediglich um 20,7 Prozent zugenommen. Der Hauptgrund für den starken Anstieg der Hilfe der Schweiz liegt in einer vom Bundesrat beschlossenen neuen Berechnung der öffentlichen Entwicklungshilfe:

- Bis 2003 meldete die Schweiz nur die Aufenthaltskosten der anerkannten Flüchtlinge für das erste Jahr. Seit 2004 verbucht sie in der öffentlichen Entwicklungshilfe die Aufwendungen für die Präsenz aller Asylbewerber aus Entwicklungsländern im ersten Aufenthaltsjahr in der Schweiz, was für 2006 165,9 Millionen Franken ausmachte.

๖ Seit 2003 umfasst die öffentliche Entwicklungshilfe der Schweiz die bilateralen Schuldenerlasse zum Nominalwert. Nach den Beschlüssen des Pariser Klubs hat die Schweiz hohe Schuldenerlasse mit dem Irak und Nigeria unterzeichnet. Diese Schuldenerleichterungen stellen aber keine effektive Ausgabe dar und haben keine Auswirkung auf den Bundeshaushalt. 2005 betrafen die wichtigsten Schuldenerleichterungen den Irak, Nigeria und die Demokratische Republik Kongo (mit jeweils 202 Millionen, 62 Millionen und 14,5 Millionen Franken im Rahmen der öffentlichen Entwicklungshilfe). 2006 betrafen die Schuldenerlasse Nigeria (mit 63,9 Millionen Franken) sowie Serbien und Montenegro (mit 58,8 Millionen Franken).

๖ Seit 2005 werden die Schenkungen aus überschüssigen Materialbeständen der Schweizer Armee ebenfalls in die öffentliche Entwicklungshilfe einbezogen, was 2006 einen Betrag von 16,2 Millionen Franken ausmachte.

๖ Als weniger umstrittenes Element wird seit 2003 ein grösserer Teil der Aktionen zur Friedensförderung und zivilen Konfliktbewältigung, die respektive 
vom Eidgenössischen Departement für Verteidigung, Bevölkerungsschutz und Sport (VBS, 2006 in Höhe von 45 Millionen Franken) und vom Eidgenössischen Departement für auswärtige Angelegenheiten (EDA, 2006 für 49,3 Millionen Franken) durchgeführt werden, in der öffentlichen Entwicklungshilfe verbucht.

\section{Grafik B.7: Entwicklung einiger Komponenten der öffentlichen Entwicklungshilfe der Schweiz, 2002-2006 (in Millionen Franken)}

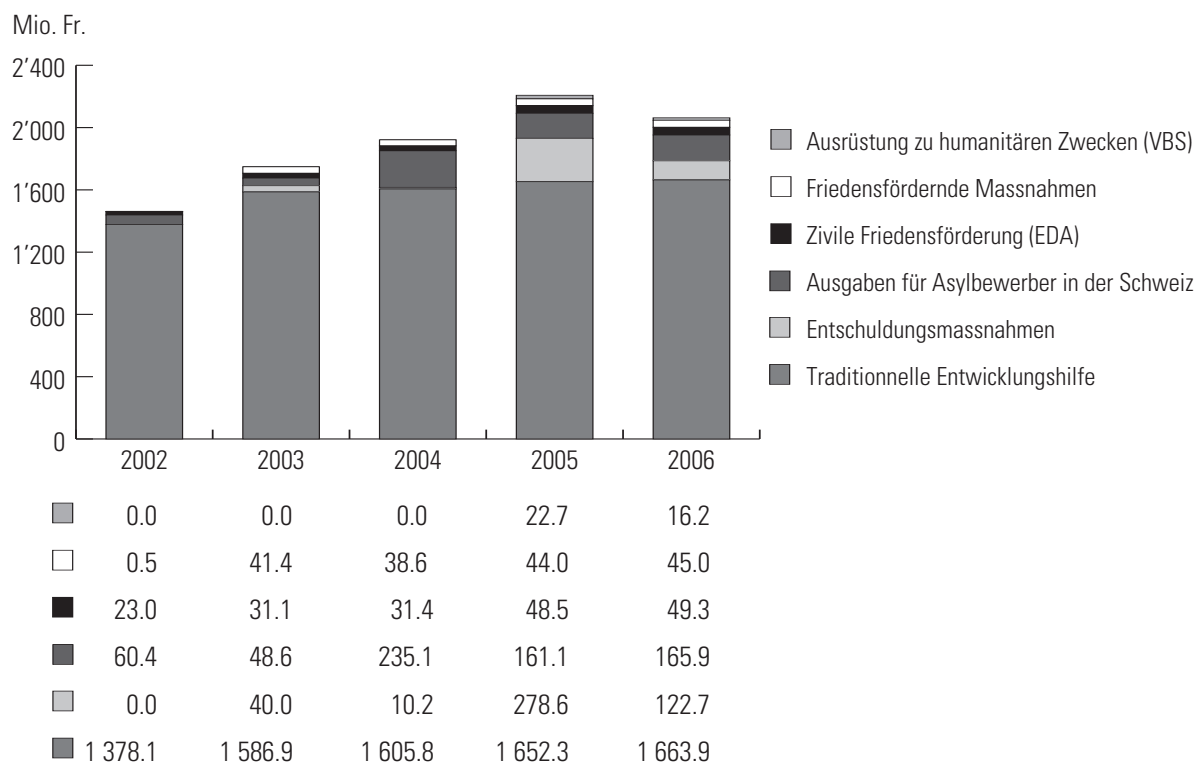

Quelle: DEZA, Schweizerische Hilfe für Entwicklungsländer und Oststaaten, Bern, DEZA, Ausgaben 2003 bis 2005. DDC, <http://www.ddc.admin.ch>>Die DEZA >Zahlen und Fakten >APD - Öffentliche Entwicklungshilfe >APD 2006. 
Die Grafik B.8 zeigt, dass die Zunahme der öffentlichen Entwicklungshilfe der Schweiz - anteilmässig am BNE - längerfristig (hier seit 1990) nicht sehr beträchtlich ist.

\section{Grafik B.8: Entwicklung der öffentlichen Entwicklungshilfe der Schweiz, 1990-2006 (in Prozent des BNE)}

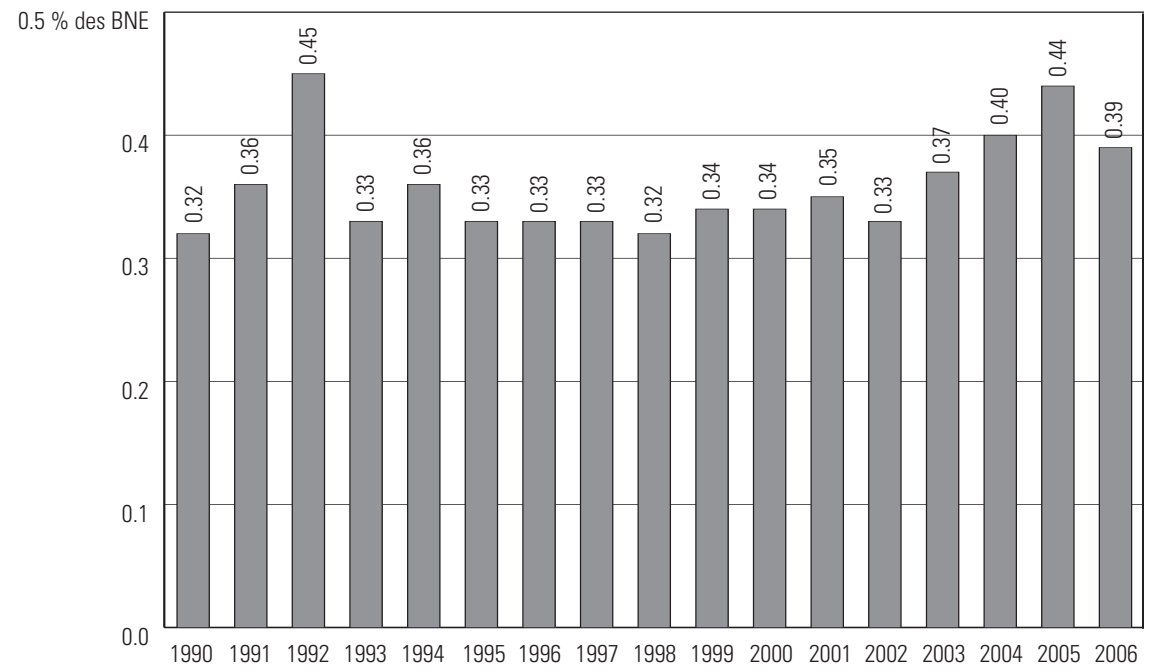

Quelle: Anhand von Daten des Statistischen Dienstes der DEZA erstellte Grafik.

\section{B.3.3. Multilaterale Hilfe}

Gemäss den von der DEZA veröffentlichten Statistiken belief sich die bilaterale Hilfe im Jahr 2006 auf 1571,5 Millionen Franken, was über drei Viertel der gesamten öffentlichen Entwicklungshilfe ausmacht. Die multilaterale Hilfe belief sich auf 491,8 Millionen Franken und machte 23,8 Prozent der öffentlichen Entwicklungshilfe aus (siehe Grafik B.9). Der Anteil der multilateralen Hilfe ist in den letzten Jahren im Anstieg begriffen.

Die allgemeinen Beiträge der Schweiz an die internationalen Organisationen sind in der multilateralen Hilfe enthalten (Beiträge in einen gemeinsamen, von der internationalen Organisation verwalteten Fonds). Jedoch wird ein bedeutender Teil der Schweizer Beiträge, die über internationale Organisationen laufen, in der bilateralen Hilfe berücksichtigt (Beiträge der Schweiz an von ihr ausgewählte Programme). Die bilaterale Hilfe für das Jahr 2006 umfasst die multibilateralen Aktionen der UN-Oganisationen (121 Millionen Franken von SECO und DEZA), gewisse Beiträge an die internationalen Finanzinstitutionen (37,6 Millionen Franken) sowie die Beiträge an andere internationale Organisationen wie das Internationale Komitee vom Roten Kreuz (IKRK), die Beratungsgruppe für internationale Agrarforschung (CGIAR) oder die Weltnaturschutzunion (IUCN) (193,8 Millionen Franken).

Mit einer multilateralen Hilfe von 491,8 Millionen Franken und spezifischen Beiträgen von 352,4 Millionen Franken wurden im Jahr 2006 somit insgesamt 
844,2 Millionen Franken an Schweizer Beiträgen über die internationalen Organisationen geleitet, was 40,9 Prozent der gesamten öffentlichen Entwicklungshilfe (2005:36\%) entspricht.

Grafik B.9: Aufteilung der öffentlichen Entwicklungshilfe der Schweiz nach bilateraler Hilfe, multilateraler Hilfe und multi-bilateraler Hilfe, 2006 (in Mio. Franken und Prozent)

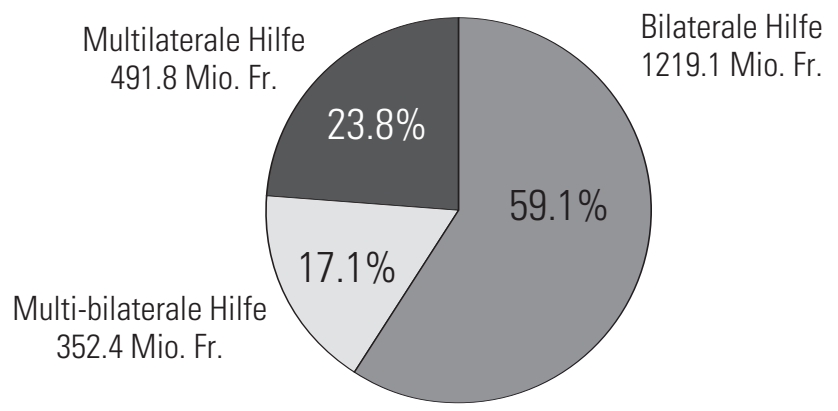

Quelle: Anhand von Daten des Statistischen Dienstes der DEZA erstellte Grafik.

Grafik B.10: Aufteilung der multilateralen öffentlichen Entwicklungshilfe der Schweiz nach Art der internationalen Organisationen, 2006 (in Millionen Franken und Prozent)

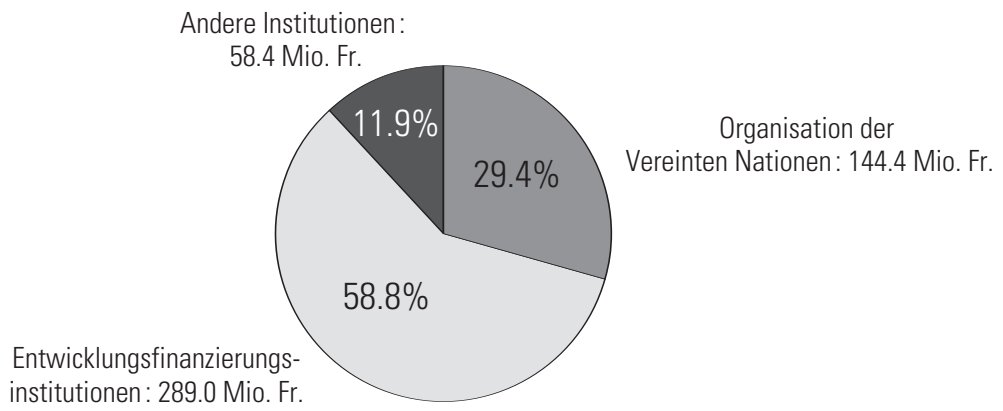

Quelle: Anhand von Daten des Statistischen Dienstes der DEZA erstellte Grafik

\section{B.3.4. Bilaterale Hilfe}

Die bilaterale Hilfe der Schweiz belief sich 2006 auf 1571 Millionen Franken. Die Grafik B.11 zeigt, dass diese Hilfe vor allem an die ärmsten Länder geht: Die am wenigsten entwickelten Länder (LDC) erhielten knapp 20 Prozent und die sonstigen einkommensschwachen Länder 15,1 Prozent. Die Länder mit mittlerem Einkommen erhielten 23,8 Prozent. 41 Prozent der bilateralen Hilfe sind geografisch nicht aufgeteilt (Hilfe über internationale Organisationen, Beiträge an das IKRK, Ausgaben in der Schweiz). 
Grafik B.11: Bilaterale öffentliche Entwicklungshilfe der Schweiz für die Entwicklungsländer, nach Ländergruppen (in Millionen Franken und Prozent)

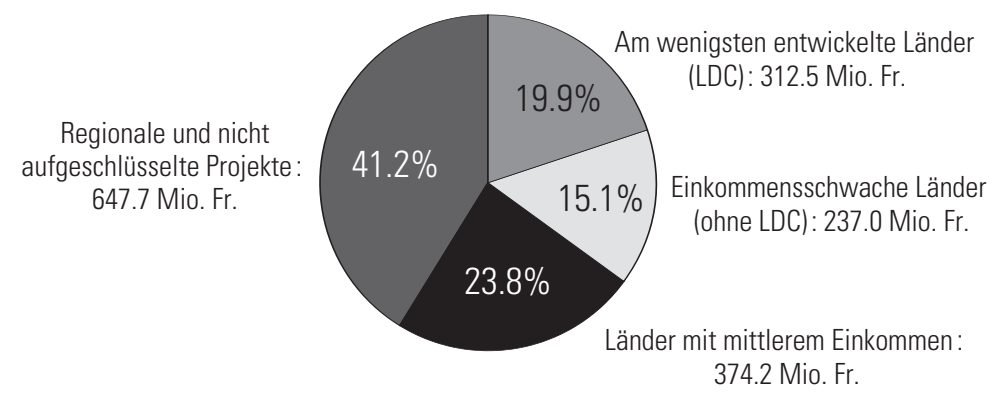

Quelle: DEZA, Entwicklungshilfe der Schweiz. Statistiken 2006, Bern, DEZA, 2008, Tabelle 3, „Contributions bilatérales: aide publique et privée par pays", und Tabelle 2, "Flux financiers bilatéraux“.

Untersucht man die bilaterale Hilfe nach Kontinenten (siehe Grafik B.12), so stellt man fest, dass die afrikanischen Länder ein Viertel und die asiatischen Länder rund 20 Prozent der Hilfe erhalten haben. 8,2 Prozent der Hilfe gingen an die lateinamerikanischen Länder und 13,3 Prozent an die Entwicklungsländer Südosteuropas.

\section{Grafik B.12: Bilaterale öffentliche Entwicklungshilfe der Schweiz für die Entwicklungs- länder, nach Kontinenten, 2006 (in Millionen Franken und Prozent)}

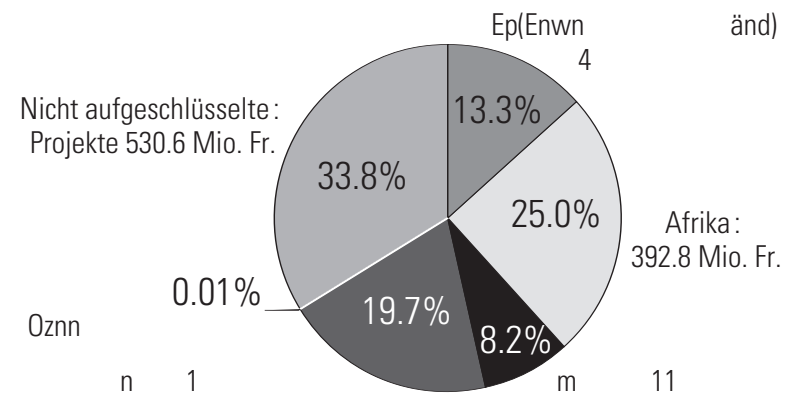

Quelle: DEZA, Entwicklungshilfe der Schweiz. Statistiken 2006, Bern, DEZA, 2008, Tabelle 3, „Contributions bilatérales: aide publique et privée par pays", und Tabelle 2, „Flux financiers bilatéraux". 
Grafik B.13: Entwicklung der privaten Hilfe der Schweizer NRO für die Entwicklungsländer, 1985-2006 (in Millionen Franken)

Mio. Fr.

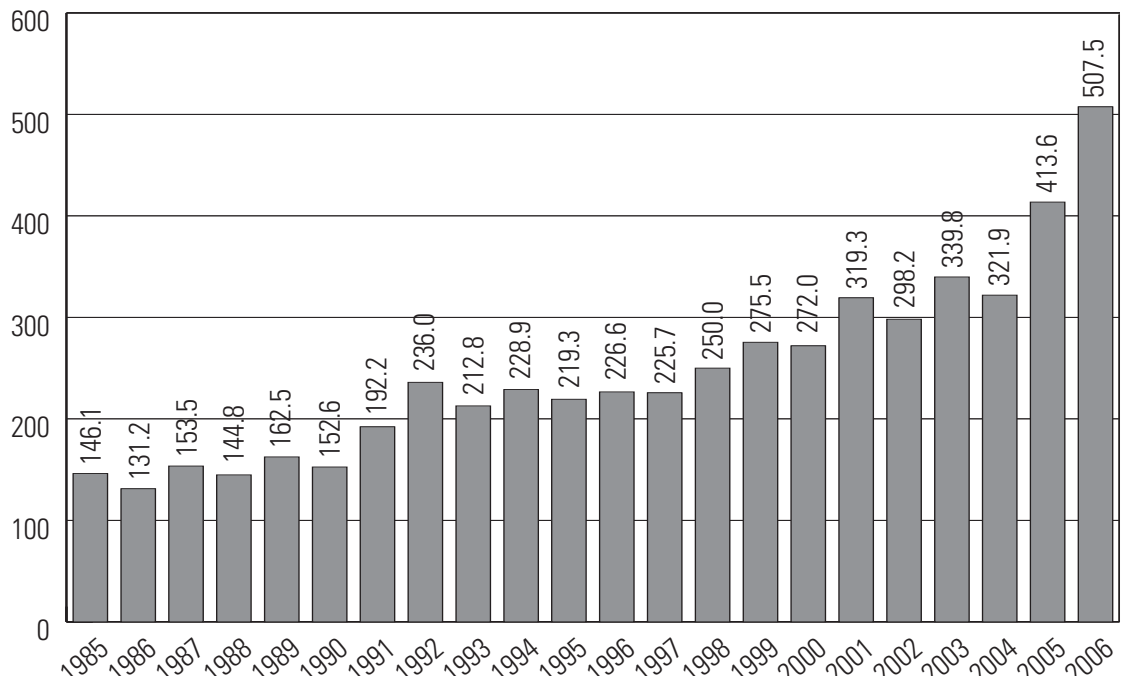

Quelle: DEZA, Hilfe der Schweiz für die Entwicklungsländer, Ausgaben verschiedener Jahrgänge.

Tabelle B.4: Private Hilfe der wichtigsten Schweizer NRO für die Entwicklungsländer, 2006 (in Tausend Franken, Anteile und kumulierte Anteile in Prozent)

\begin{tabular}{lcrr}
\hline Organisation & Tausend Franken & $\%$ & $\sum \%$ \\
\hline Caritas - Schweiz & $47^{\prime} 633$ & 9.4 & 9.4 \\
\hline Schweizerisches Rotes Kreuz (SRK) & $44^{\prime} 771$ & 8.8 & 18.2 \\
\hline Ärzte ohne Grenzen (MSF) & $42^{\prime} 549$ & 8.4 & 26.6 \\
\hline World Vision Schweiz & $30^{\prime} 561$ & 6.0 & 32.6 \\
\hline MEDAIR & $28^{\prime} 040$ & 5.5 & 38.1 \\
\hline Stiftung Kinderhospital Kantha Bopha (Kambodscha) & $24^{\prime} 742$ & 4.9 & 43.0 \\
\hline Stiftung Terre des hommes (Lausanne) & $24^{\prime} 551$ & 4.8 & 47.9 \\
\hline Hilfswerk der Evangelischen Kirchen der Schweiz (HEKS) & $24^{\prime} 163$ & 4.8 & 52.6 \\
\hline Helvetas & $13^{\prime} 972$ & 2.8 & 55.4 \\
\hline Schweiz. Freunde der SOS Kinderdörfer & $9^{\prime} 051$ & 1.8 & 57.1 \\
\hline Fondation Limmat & $9^{\prime} 042$ & 1.8 & 58.9 \\
\hline Limmat Stiftung & $9^{\prime} 042$ & 1.8 & 60.7 \\
\hline Kinderhilfe Bethlehem & $8^{\prime} 284$ & 1.6 & 62.3 \\
\hline Schweizerisches Komitee für UNICEF & $7^{\prime} 710$ & 1.5 & 63.9 \\
\hline Fastenopfer & $7^{\prime} 157$ & 1.4 & 65.3 \\
\hline Stiftung Novartis & $6^{\prime} 035$ & 1.2 & 66.5 \\
\hline Schweiz. Tropeninstitut & $6^{\prime} 017$ & 1.2 & 67.6 \\
\hline Bethlehem Mission Immensee & $5^{\prime} 795$ & 1.1 & 68.8 \\
\hline Schweizerisches Arbeiterhilfswerk (SAH) & $5^{\prime} 655$ & 1.1 & 69.9 \\
\hline Swisscontact & $5^{\prime} 508$ & 1.1 & 71.0 \\
\hline MFM Stiftung Äthiopienhilfe & $4^{\prime} 800$ & 71.9 \\
\hline Leprahilfe Emmaüs Schweiz & $4^{\prime} 706$ & 0.9 & 72.9 \\
\hline Hilfsorganisation HMK & $4^{\prime} 339$ & 0.9 & 73.7 \\
\hline Stiftung Kinderdorf Pestalozzi & $4^{\prime} 159$ & 0.9 & 74.5 \\
\hline
\end{tabular}




\begin{tabular}{|c|c|c|c|}
\hline Organisation & Tausend Franken & $\%$ & $\sum \%$ \\
\hline Cœurs pour tous & 4 4'000 & 0.8 & 75.3 \\
\hline Fondation Hirondelle & $3{ }^{\prime} 970$ & 0.8 & 76.1 \\
\hline Benediktiner Uznach & 3906 & 0.8 & 76.9 \\
\hline Mission 21 & 3'821 & 0.8 & 77.6 \\
\hline Terre des Hommes Schweiz (Genf) & $3^{\prime} 450$ & 0.7 & 78.3 \\
\hline Handicap International & $3^{\prime} 363$ & 0.7 & 79.0 \\
\hline Heilsarmee & 3306 & 0.7 & 79.6 \\
\hline Vereinigung Don Bosco Werk & $3^{\prime} 120$ & 0.6 & 80.2 \\
\hline Christoffel Blindenmission - CBM & $2 ' 918$ & 0.6 & 80.8 \\
\hline WWF Schweiz & $2 ' 705$ & 0.5 & 81.3 \\
\hline Nouvelle Planète & $2 ' 664$ & 0.5 & 81.9 \\
\hline Swissaid & $2 ' 599$ & 0.5 & 82.4 \\
\hline Fondation Pro Victimis & $2 ' 589$ & 0.5 & 82.9 \\
\hline Terre des hommes (VS) & $2 ' 512$ & 0.5 & 83.4 \\
\hline Gesamtbetrag für obige $38 \mathrm{NRO}^{\circ}$ & $423^{\prime} 205$ & 83.4 & \\
\hline Betrag für 295 weitere NRO & $84^{\prime} 304$ & 16.6 & \\
\hline Gesamtbetrag & $507 ' 509$ & 100.0 & \\
\hline
\end{tabular}

Quelle: DEZA, Entwicklungshilfe der Schweiz. Statistiken 2006, Bern, DEZA, 2008, Tabelle 6.

a Berücksichtigt wurden die NRO, die mehr als 2,5 Millionen Franken aus Eigenmitteln für die Hilfe bereitstellten.

Die Angaben über die private Hilfe der schweizerischen Nichtregierungsorganisationen betreffen die Beträge, welche durch eigene Mittel der NRO finanziert werden (Beiträge und Spenden von Mitgliedern, Erträge aus Sammelkampagnen, Eigenfinanzierung, Erlöse aus Verkäufen). Diese Beträge haben sich in den letzten Jahren regelmässig erhöht, wobei sie von 146,1 Millionen Franken für 1985 auf 225,7 Millionen Franken für 1997 anstiegen und sich 2006 mit 507,5 Millionen Franken noch mehr als verdoppelt haben (siehe Grafik B.13). Zwischen 1996 und 2006 sind die Leistungen der NRO von 0,056 Prozent auf 0,096 Prozent des BNE angestiegen.

Neben den 507,5 Millionen Franken an privaten Mitteln, die für Projekte in den Entwicklungsländern eingesetzt werden, haben die NRO auch Beiträge vom Bund, von Kantonen und Gemeinden für die Verwaltung von Projekten erhalten. So zahlte die DEZA beispielsweise 221,1 Millionen Franken für Projekte der Entwicklungszusammenarbeit oder für humanitäre Einsätze an die NRO aus und das SECO stellte 5,8 Millionen Franken bereit.

Tabelle B.4 führt die privaten Spenden der wichtigsten schweizerischen Nichtregierungsorganisationen auf. In der Schweiz gibt es neben einigen grossen NRO auch eine Vielzahl kleiner Organisationen. Im Jahr 2006 haben sich die 38 grössten Organisationen 83,4 Prozent der privaten Mittel für Projekte geteilt. Rund 300 weitere erfasste NRO verwalteten die restlichen 16,6 Prozent.

Tabelle B.5 zeigt die Entwicklung der Hilfe der NRO für die 30 wichtigsten Empfängerländer (aus Eigenmitteln der NRO, ohne öffentlliche Beiträge finanzierte Projekte). In den letzten Jahren haben die Hilfeleistungen der NRO für bestimmte, von Naturkatastrophen betroffene Gebiete und für Länder mit Konfliktsituationen stark zugenommen. Die aussergewöhnlichen Spendeneingänge nach dem Tsunami, der mehrere asiatische Länder im Dezember 2004 heimgesucht hatte, ermöglichten es den NRO, vor allem in Indonesien und Sri Lanka zahlreiche Wiederaufbauprojekte zu finanzieren. Die Hilfe der NRO für diese beiden Länder ist somit von 3,9 Millionen Franken im Jahr 2004 auf 40,4 Millionen Franken für 2005 und 60,3 Millionen Franken für 2006 angestiegen. 
Die Hilfe der NRO konzentriert sich noch stärker als die öffentliche Entwicklungshilfe auf die ärmsten Länder: 2006 haben die am wenigsten entwickelten Länder 38,8 Prozent und die sonstigen einkommensschwachen Länder 16,1 Prozent der Leistungen der NRO erhalten. 36,4 Prozent der Hilfe ging an Länder mit mittlerem Einkommen (8.8\% Regionalprojekte).

Tabelle B.5: Entwicklung der Hilfe der NRO nach Regionen und Hauptempfängerländern, 2001-2006 (in Millionen Franken)

\begin{tabular}{|c|c|c|c|c|c|c|}
\hline Land & 2001 & 2002 & 2003 & 2004 & 2005 & 2006 \\
\hline Afrika & 98.0 & 100.5 & 122 & 112.3 & 133.75 & 179.2 \\
\hline davon Sudan & 10.7 & 9.1 & 10.6 & 11.8 & 14.7 & 23.1 \\
\hline Kongo, Dem. Rep. & 4.5 & 5.5 & 9.2 & 5.2 & 8.5 & 20.6 \\
\hline Äthiopien & 5.9 & 5.5 & 9.6 & 8.5 & 12.6 & 14.8 \\
\hline Tansania & 6.7 & 7.7 & 6.4 & 6.2 & 8.6 & 12.3 \\
\hline Uganda & 3.1 & 4.2 & 4.0 & 4.6 & 3.6 & 8.4 \\
\hline Kamerun & 3.8 & 3.4 & 5.9 & 4.6 & 6.4 & 7.7 \\
\hline Somalia & 1.6 & 1.4 & 2.7 & 2.5 & 3.1 & 6.6 \\
\hline Mosambik & 11.0 & 6.0 & 7.1 & 5.3 & 6.7 & 6.5 \\
\hline Kenia & 4.0 & 4.7 & 3.1 & 2.4 & 3.8 & 6.2 \\
\hline Niger & 0.8 & 1.1 & 1.3 & 1.3 & 13.0 & 5.7 \\
\hline Angola & 2.4 & 3.5 & 8.1 & 2.5 & 3.1 & 5.6 \\
\hline Liberia & 0.0 & 1.0 & 1.7 & 4.0 & 0.9 & 4.2 \\
\hline Mali & 2.7 & 3.6 & 6.7 & 5.3 & 5.0 & 4.0 \\
\hline Amerika & 65.0 & 62.5 & 56.0 & 55.8 & 55.5 & 71.7 \\
\hline davon Kolumbien & 6.9 & 5.6 & 5.2 & 5.7 & 6.2 & 11.0 \\
\hline Brasilien & 8.5 & 9.0 & 8.2 & 8.3 & 8.5 & 9.4 \\
\hline Bolivien & 5.6 & 8.8 & 5.4 & 5.3 & 6.5 & 8.4 \\
\hline Peru & 5.5 & 5.1 & 4.9 & 4.6 & 4.6 & 8.0 \\
\hline Guatemala & 4.3 & 5.3 & 4.8 & 4.1 & 5.9 & 6.6 \\
\hline Nicaragua & 5.9 & 5.4 & 4.8 & 5.1 & 3.5 & 5.6 \\
\hline Haiti & 3.9 & 3.5 & 3.9 & 5.5 & 5.3 & 4.8 \\
\hline Asien & 89.7 & 90.9 & 111.4 & 106.9 & 175.3 & 199.5 \\
\hline davon Sri Lanka & 1.7 & 1.2 & 2.0 & 1.8 & 24.1 & 37.6 \\
\hline Kambodscha & 20.0 & 20.2 & 19.8 & 22.8 & 32.9 & 27.9 \\
\hline Indien & 21.6 & 23.6 & 20.1 & 16.0 & 31.9 & 27.2 \\
\hline Indonesien & 1.9 & 1.2 & 1.8 & 2.1 & 16.3 & 22.7 \\
\hline Pakistan & 1.4 & 1.1 & 0.4 & 0.3 & 7.5 & 13.5 \\
\hline $\begin{array}{l}\text { Gebiete unter palästinensischer } \\
\text { Verwaltung }\end{array}$ & 1.7 & 1.5 & 9.1 & 11.6 & 11.3 & 10.8 \\
\hline Afghanistan & 5.1 & 7.6 & 10.4 & 7.2 & 3.2 & 6.5 \\
\hline Iran & 0.0 & 0.0 & 0.0 & 6.2 & 6.1 & 5.9 \\
\hline Vietnam & 3.8 & 5.2 & 5.3 & 4.6 & 5.0 & 5.5 \\
\hline Philippinen & 5.2 & 7.0 & 6.6 & 4.4 & 5.6 & 4.6 \\
\hline Nepal & 1.4 & 1.8 & 7.6 & 3.5 & 3.8 & 4.0 \\
\hline Europa (Hilfeempfängerländer) & 45.6 & 19.3 & 18.7 & 29.4 & 21.1 & 18.1 \\
\hline davon Bosnien-Herzegowina & 4.8 & 3.6 & 4.2 & 7.9 & 7.5 & 6.0 \\
\hline Serbien-und-Montenegro & 27.2 & 7.6 & 2.1 & 11.1 & 7.2 & 4.4 \\
\hline Hilfe der NRO insgesamt & 319.3 & 298.2 & 339.8 & 321.9 & 413.6 & 507.5 \\
\hline
\end{tabular}

Quelle: DEZA, Hilfe der Schweiz für die Entwicklungsländer, verschiedene Jahrgänge.

a Die Tabelle führt die Entwicklung jener Zahlungen auf, die für 2005 oder 2006 höher als 4 Millionen Franken waren. 